\title{
Effect of Specific Music on Psychoneuroimmunological Responses
}

\author{
Abdul Waheed ${ }^{1 *}$, David Kossor ${ }^{2}$, William Collins ${ }^{3}$ and Amy Camie ${ }^{4}$ \\ ${ }^{1}$ Biochemistry and Molecular Biology, St. Louis University, USA \\ ${ }^{2}$ Principal/CEO, Syncratic Therapies LLC, Charlotte, USA \\ ${ }^{3}$ Principal/Owner, Reintegrative Health Institute, St. Louis University, USA \\ ${ }^{4}$ Composer, Professional Harpist, St. Louis, USA
}

*Corresponding author: Abdul Waheed, Biochemistry and Molecular Biology, St. Louis University, Doisy Research Center, 1100 S. Grand Blvd., 615, St. Louis, MO 63104, USA, Tel: 314-977-9274;

Amy Camie, Composer, Professional Harpist, St. Louis, Missouri, 63146, USA, Tel: 314-603-4430

\begin{abstract}
A pilot study was done to measure quantitative EEG (qEEG) brainwave frequencies and behavioral indices of five women undergoing chemotherapy treatment for cancer, before and after listening to specific music (The Magic MirrorInspired Reflections solo harp CD written, performed and recorded by Amy Camie) once a day for ten days. Following treatments, the EEG profile for all five participants improved and subjective reports from daily questionnaires confirmed a positive effect of the music.

A further proof of principle investigation of potential effects of listening to The Magic Mirror music on physiological and psychological stress and immunoreactivity was done in ten healthy individuals by measuring biomarkers of physiological and psychological stress (carbonic anhydrase VI (CAVI), IgA, IgG, and ó-amylase) in saliva samples that were collected from each individual before and after listening to the 23-minute CD. Additional samples were collected 24 hours later. Secretory $\lg A$, IgG, and CAVI biomarkers were increased in saliva and remained high until 24 hours after music, suggesting The Magic Mirror produced measurable effects on immune response in individuals.

A second proof of principle study looked at the duration of the effects. Similar to the first study, the second study was done repeating the same study design, where levels of secretory $\lg \mathrm{A}, \lg \mathrm{G}$, ó-amylase, and $\mathrm{CAVI}$ in saliva samples again were shown to increase during and after listening, and these changes persisted for 2-3 days. These results suggest that listening to The Magic Mirror CD produces neurosecretory responses indicative of reduced physiological and psychological stress and may be a useful adjunctive therapy for cancer or physiologically stressed patients.
\end{abstract}

\section{Keywords}

Psychoneuroimmunology, Immune system, Music, Stress, Brainwaves, Cancer, Chemo brain, Cognitive deficits, Cancer and treatment-related neurocognitive dysfunction

\section{Abbreviations}

PNI: Psychoneuroimmunology; qEEG: Quantitative Electroencephalogram; CAVI: Carbonic Anhydrase VI; CRND: Cancer and Treatment-Related Neurocognitive Dysfunction; TBI: Traumatic Brain Injury; BEHAVE-AD: Behavioral Pathology in Alzheimer's Disease Rating Scale; ACTH: Adrenocorticotropic Hormone; CRH: CorticotropinReleasing Hormone

\section{Introduction}

Scientific research into the effects of music increases with the ability to accurately measure the physiology of the listener. Researchers have now indicated that music without lyrics is effective for pain management, [1] listening to yoga music at bedtime is good for the heart, [2] and music, regardless of the listener's personal preference, can be sensed at an unconscious level affecting autonomic cardiovascular responses [3].

Musical selections used in research are often generalized into genres such as classical or relaxing without due consideration of the performer or the intent of the composer.

Musicians are able to express and communicate their intentions to listeners through variables that collective-

Citation: Waheed A, Kossor D, Collins W, Camie A (2018) Effect of Specific Music on Psychoneuroimmunological Responses. Int J Oncol Res 1:011.

Accepted: December 29, 2018; Published: December 31, 2018

Copyright: (C) 2018 Waheed A, et al. This is an open-access article distributed under the terms of the Creative Commons Attribution License, which permits unrestricted use, distribution, and reproduction in any medium, provided the original author and source are credited. 
ly create the listening experience such as tempo, dynamics, rhythm, and the unique characteristics of their instrument(s) [4]. Individual differences in performers' personality traits, ability to experience empathy, musical background and proficiency in their preferred genre of music, all affect their ability to communicate emotional expressions [4].

To contribute to the gap in knowledge surrounding intention between composer, performer, listener, and measureable results, The Magic Mirror-Inspired Reflections by Amy Camie, was chosen for the pilot studies referenced in this article. As the composer and performer, Camie, could potentially offer additional insights into any measureable effects within the listener, potentially expanding the gap of knowledge surrounding intention.

Acute stress can be studied in relationship to activation of the hypothalamic-pituitary-adrenal axis. Bear, Connors and Paradiso [5] outline this axis as follows. The amygdala sends projections to parts of the brain that react to adverse stimuli. These include portions of the lower brainstem that are involved in controlling the autonomic nervous system and the nucleus of the hypothalamus. The hypothalamus then releases a peptide called corticotropin-releasing hormone $(\mathrm{CRH})$ into the blood. $\mathrm{CRH}$ travels a short distance to the anterior pituitary, which within seconds stimulates the release of adrenocorticotropic hormone (ACTH). ACTH enters the general circulation and travels to the adrenal cortex where it stimulates cortisol release [5].

Cortisol elevation and cancer have been studied by Van der Pompe, Antoni, and Heijnen [6]. They found that cancer patients had significant elevations in basal cortisol levels compared to controls and that metastatic breast cancer patients had higher cortisol levels than early stage breast cancer patients [6]. Utilizing a cognitive-behavioral stress management protocol, Gruess, et al. [7] noted a decrease in serum cortisol levels in stage I and II breast cancer subjects [7]. In another study by Gruess, et al. [8] salivary cortisol levels were significantly reduced as a result of relaxation training [8].

Electroencephalographic (EEG) data is another way to measure stress. EEG activity has been classified into frequency bands. The $12.5-30 \mathrm{~Hz}$ band is referred to as the beta rhythm. Beta waves are associated with a normal waking state of consciousness that range from alert focused attention to agitated anxiety. High beta waves have been correlated with salivary cortisol levels indicative of chronic stress [9].

The psychological stress of a medical diagnosis followed by the physiological stress of treatments has been shown to negatively impact the immune system [10]. Cancer and treatment-related neurocognitive dysfunction (CRND), also known as chemo brain is a side effect reportedly experienced by up to $75 \%$ of patients who have received chemotherapy treatment, with some patients reporting the experience of CRND for up to 20 years after exposure to chemotherapy $[11,12]$. Patients with CRND experience symptoms that affect their quality of life including fatigue, anxiety, depression, sleep problems, pain, inability to focus and immune responses such as inflammation [12].

The use of music is strongly supported in conjunction with cancer care, pain management, anxiety relief, depression and fatigue $[13,14]$.

In a systematic review of studies exploring the pyschoneuroimmulogical effects of music by Fancourt $D$, et al. [15], twenty-eight studies measured the psychological impact of music. Only one study explored the sustained impact of listening to familiar music on changes in psychological states of patients. After 10 weeks of listening to recorded music, improvements, as measured by the Behavioral Pathology in Alzheimer's Disease Rating Scale (BEHAVE-AD), persisted for 3 weeks [15].

To address specific cancer-related side effects such as memory loss, nausea, and mouth and throat problems, [16] proof of principle saliva sample pilot studies were conducted to determine the utility of specific salivary proteins, such as carbonic anhydrase $\mathrm{VI}$ (gustin) as a biomarker for stress in a developing larger study. To demonstrate the feasibility of including carbonic anhydrase $\mathrm{VI}$, it was decided to also measure IgA, IgG and amylase as additional salivary biomarkers of the stress response in healthy individuals [17].

Carbonic anhydrases are a family of zinc-dependent enzymes, that all catalyze the reversible hydration of carbon dioxide, to produce bicarbonate. While at least 12 specific isoforms of carbonic anhydrase have been identified in mammalian cells to-date, most of these are located within the interior of the cells, and there is only one isozyme, carbonic anhydride VI (CAVI), that is excreted from the cells, and is recovered in saliva, plasma, and other body fluids [18]. Before the enzymatic activity of CAVI was appreciated, earlier studies had isolated this zinc-binding protein as a trophic factor in saliva, where it promoted the growth and maintenance of taste buds and immunity for the oral mucosa [19]. At the time, this trophic factor was given the name "gustin", and it wasn't until several years later when its identity as CAVI was revealed [20].

Importantly, the mechanism for the trophic actions on the oral mucosa is believed to be due, in part, to the presence of zinc that is transferred to the tissues when needed, to exert numerous pleitropic roles, such as the acceleration of wound healing and to restore the sense of taste. For these reasons, CAVI was included with the battery of salivary protein biomarkers, to provide an additional indicator of innate immune function. 


\section{Materials and Methods}

\section{QEEG analyses}

A Request for Volunteers was shared with people in the community who worked with women ages $20+$ undergoing chemotherapy treatment for cancer. Five subjects were enlisted in the qEEG pilot study. Quantitative EEG (qEEG) is a method of analyzing the electrical activity of the brain to derive quantitative patterns that may correspond to diagnostic information and/or cognitive deficits.

Each subject was given a qEEG pre and post treatment. After the pre qEEG they were instructed to listen to The Magic Mirror solo harp CD once a day for ten consecutive days on a portable CD player with headphones provided to them. They were also given a Daily Checklist with specific instructions and questions that included:

Please listen to this $C D$ during the morning, afternoon or early evening and NOT right before bedtime. This CD runs 23 minutes.

\section{At what time did you listen?}

Were you sitting or lying down?

How did you feel before listening?

How did you feel afterwards?
At the end of the ten-day listening period each participant returned to the office with their Daily Checklist, CD player, and headphones. A post qEEG was done [21].

The qEEG data was obtained via nineteen electrodes placed on the scalp. The data was recorded using Deymed acquisition hardware and then processed with NeuroGuide, a normative data base developed by Dr. Robert Thatcher [22]. Each subject's data was looked at in terms of the degree to which it deviated from the norm (Figure 1). EEG frequencies (delta to beta), asymmetry and the TBI Index (traumatic brain injury) are ways of measuring cortical efficiency and information processing.

\section{Salivary biomarker analyses}

To study the immune parameters in psychoneuroimmunological responses of listening to The Magic Mirror $C D$, we chose to measure secretory IgA [23] and IgG [24] levels in saliva. We also measured salivary $\alpha$-amylase which has been used as a biomarker for physiological and psychological stress [25]. Carbonic anhydrase VI (CAVI), a secretory $\mathrm{Zn++}$ containing glycoprotein, has been reported to show a correlation between dental conditions and CAVI levels in saliva [26]. We measured all biomarkers before and after music as proof of principle to determine the viability of CAVI as a biomarker for stress.

\section{\begin{tabular}{llllllll}
\hline-3 & -2 & -1 & 0 & 1 & 2 & 3
\end{tabular}}

\section{Color chart}

Red = significant deviation from the norm

Green $=$ a statistically positive change or normalization .

TBI Index (Mild Traumatic Brain Index)

\begin{tabular}{|l|l|l|l|l|l|}
\hline Subject & Delta & Theta & Beta & High Beta & TBI Index \\
\hline 1-Pre & elevated & deficit & elevated & elevated & No \\
\hline 1-Post & reduced & improved & normalized & reduced & No \\
\hline & & & & & \\
\hline 2-Pre & elevated & elevated & deficit & elevated & Yes \\
\hline 2-Post & reduced & normalized & improved & same & $\begin{array}{l}\text { No } \\
\text { change }\end{array}$ \\
\hline & & & & & \\
\hline 3-Pre & & & elevated & elevated & Yes \\
\hline 3-Post & & & reduced & reduced & improved \\
\hline & & & & & \\
\hline 4-Pre & & deficit & deficit & deficit & Yes \\
\hline 4-Post & & normalized & normalized & normalized & improved \\
\hline & & & & & (nes \\
\hline 5-Pre & & deficit & deficit & elevated & Yes \\
\hline 5-Post & & normalized & normalized & reduced & $\begin{array}{l}\text { No } \\
\text { change }\end{array}$ \\
\hline
\end{tabular}

Figure 1: Overview of pre and post qEEG measurements of five subjects after listening to The Magic Mirror CD once a day for 10 days. 


\section{Z Scored FFT Summary Information}
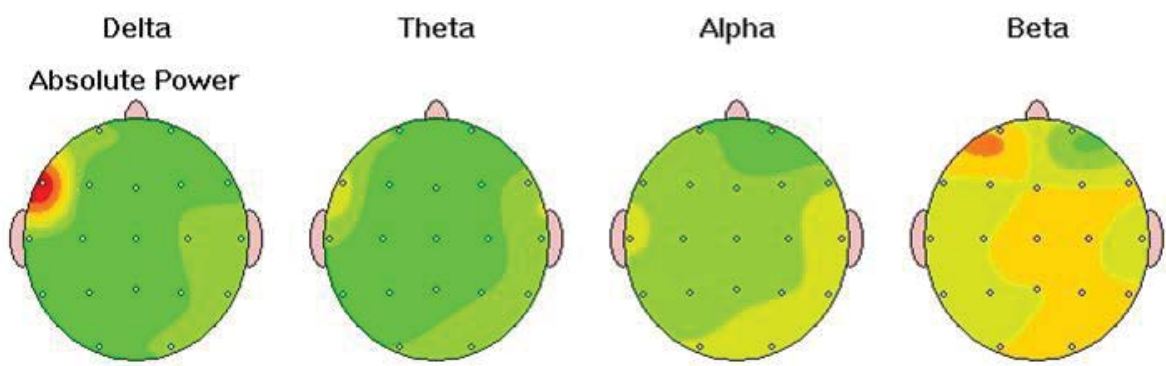

High Beta

Relative Power
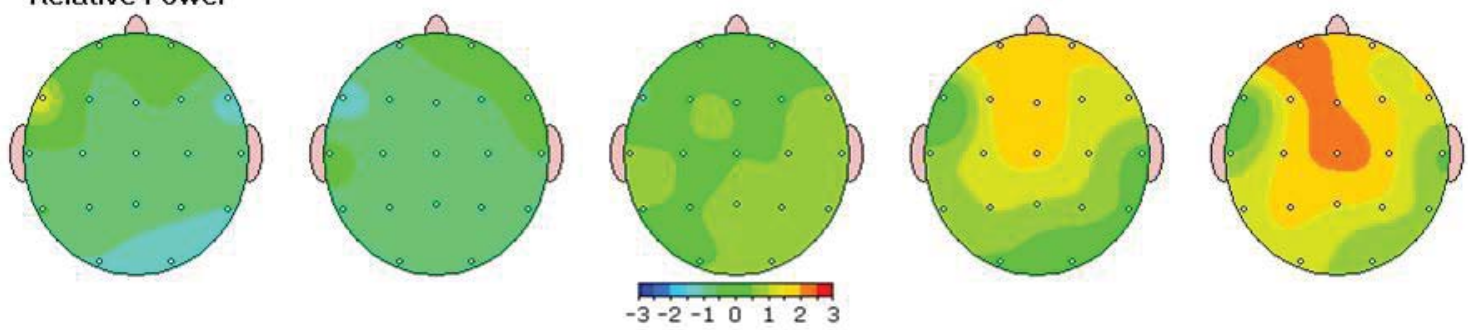

\section{Amplitude Asymmetry}

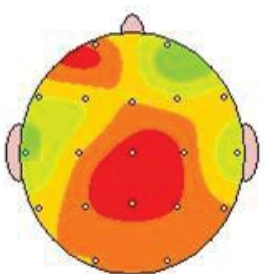

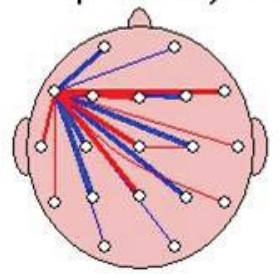
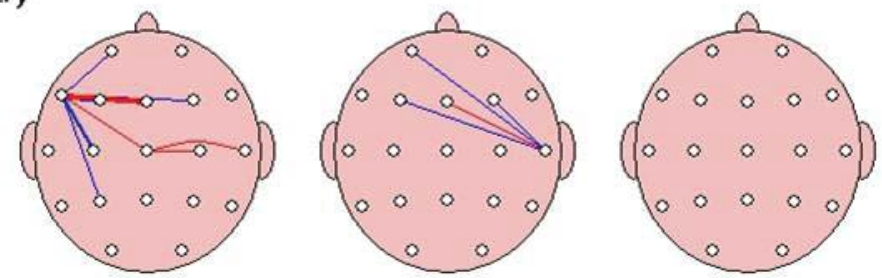

Figure 1A: Overview of subject's pre qEEG before listening to The Magic Mirror CD.

Montage: LinkEars

\section{Z Scored FFT Summary Information}

Delta

Absolute Power

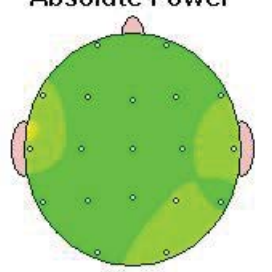

Relative Power

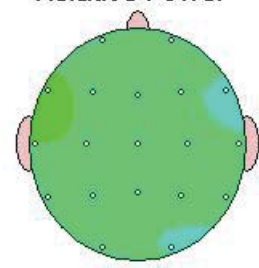

Amplitude Asymmetry
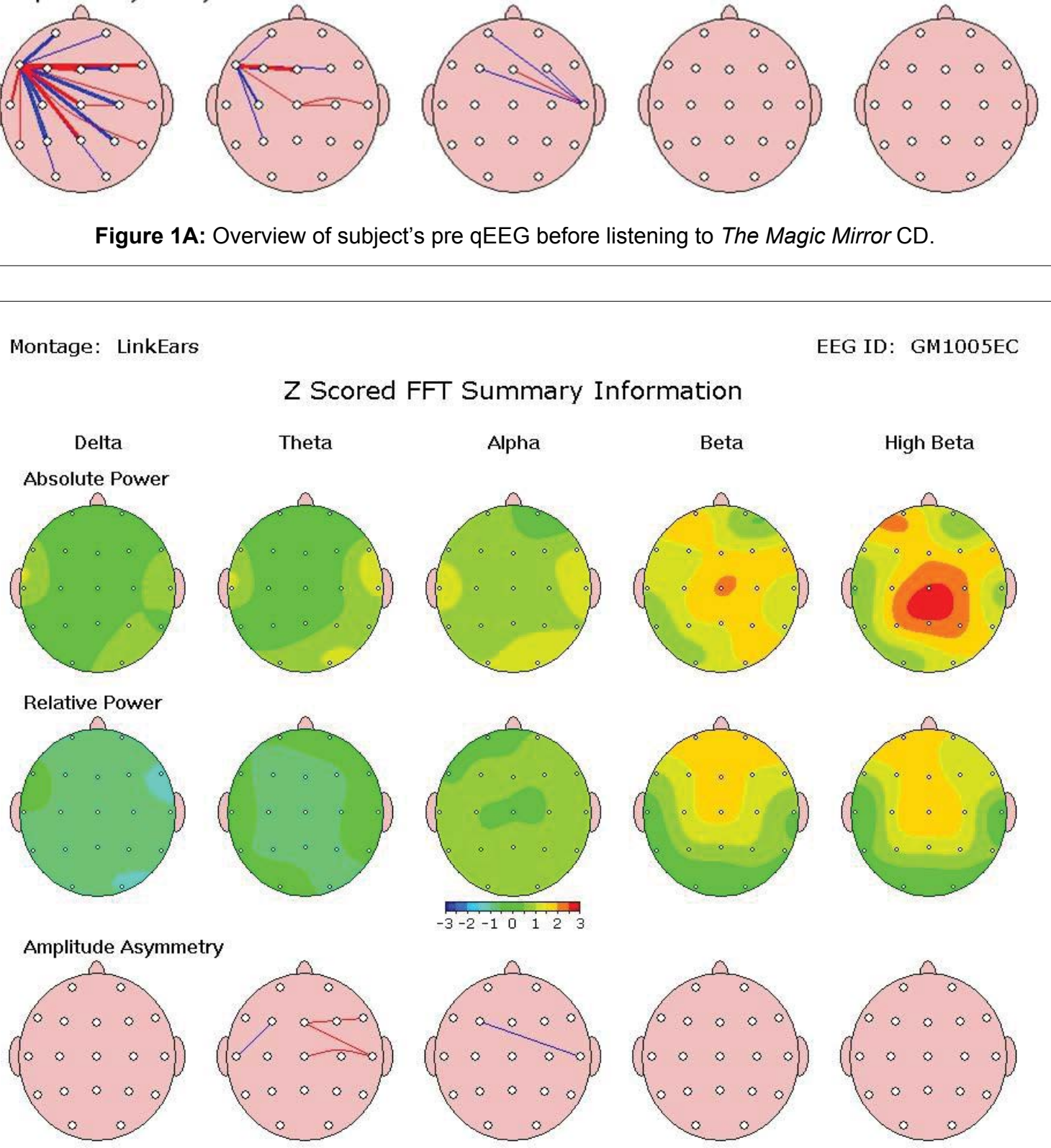

Figure 1B: Overview of subject's post qEEG after listening to The Magic Mirror CD once a day for 10 days. 

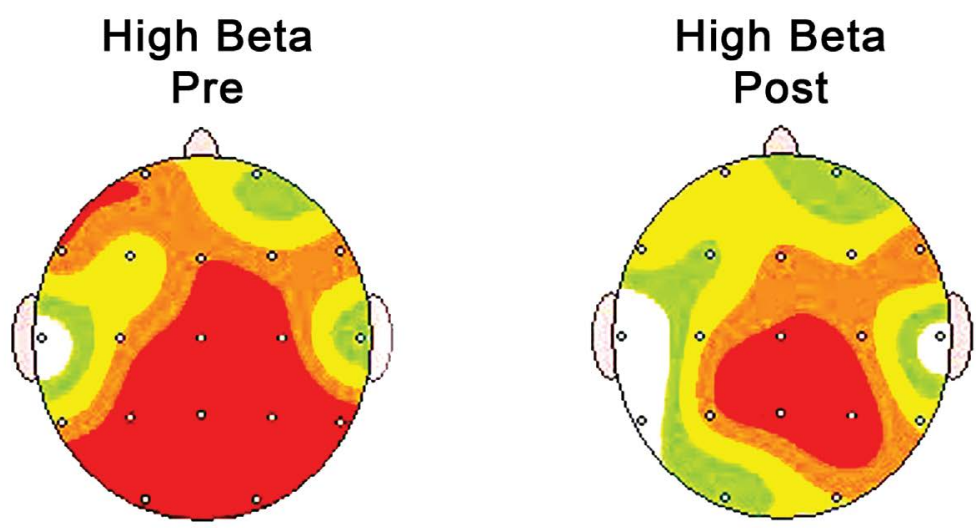

Figure 1C: Subject 1 decrease in high beta frequency after listening to The Magic Mirror CD once a day for 10 days.
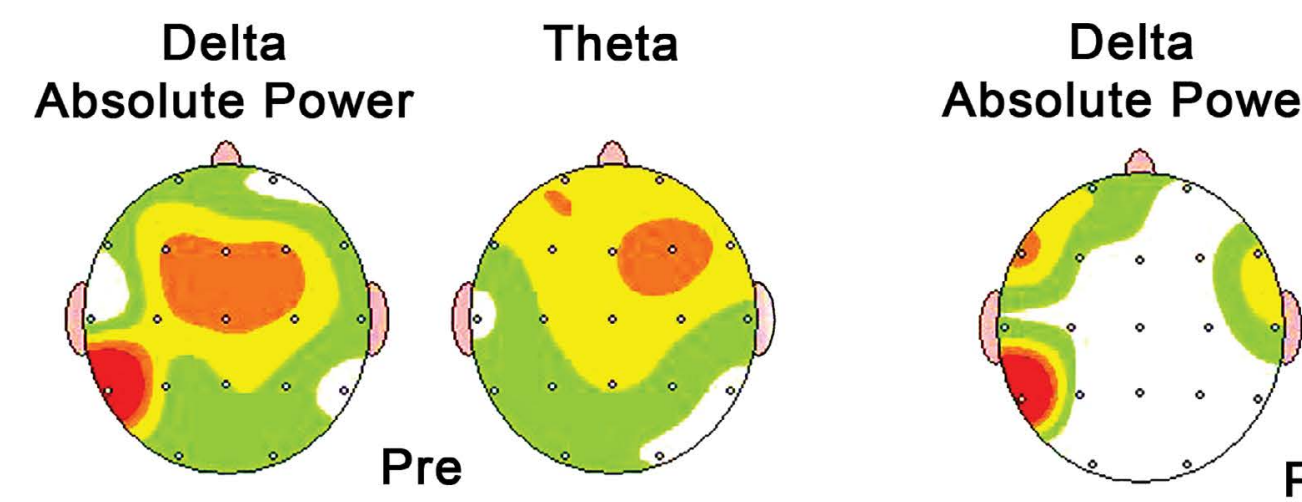

Theta

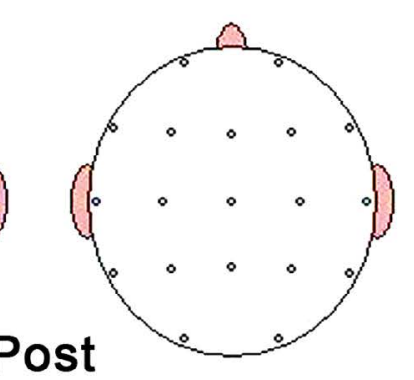

Figure 1D: Subject 2 decrease in delta and theta frequencies after listening to The Magic Mirror CD once a day for 10 days.
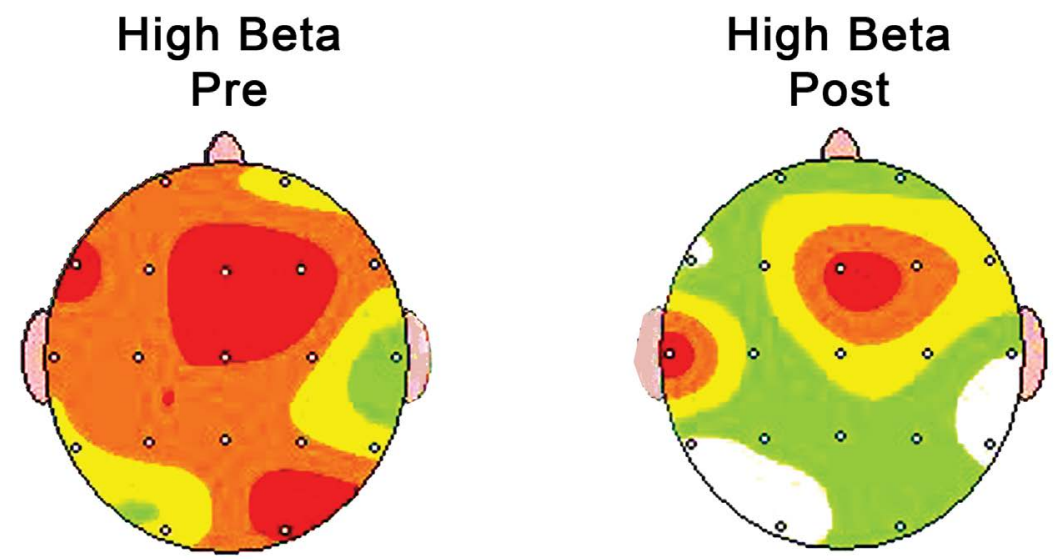

Figure 1E: Subject 3 decrease in high beta frequency after listening to The Magic Mirror CD once a day for 10 days.

In Study 1, all individuals who were able to consent were invited to participate, regardless of gender or age. Eight healthy individuals, ages 8-65 years of age, participated. Everyone met in the home of one of the participants. A baseline saliva sample was collected from each participant by placing an absorbent cotton ball in the mouth until it became saturated with saliva and was then transferred to a polyethylene tube and stored refrigerated $\left(2-8{ }^{\circ} \mathrm{C}\right)$. The intervention was the 23-minute solo harp CD, The Magic Mirror. Each individual moved to a separate room in the house and listened with headphones on their own $C D$ player. A second saliva sample was collected immediately after listening to the music. At approximately the same time of day, 24 hours later, another saliva sample was collected.

In Study 2, seven participants from the first study and two additional individuals participated. The initial study design was repeated with additional saliva samples collected 48 hours and 72 hours post listening.

Rabbit antihuman amylase anti IgG-FC, and anti- 


\section{Delta Absolute Power}
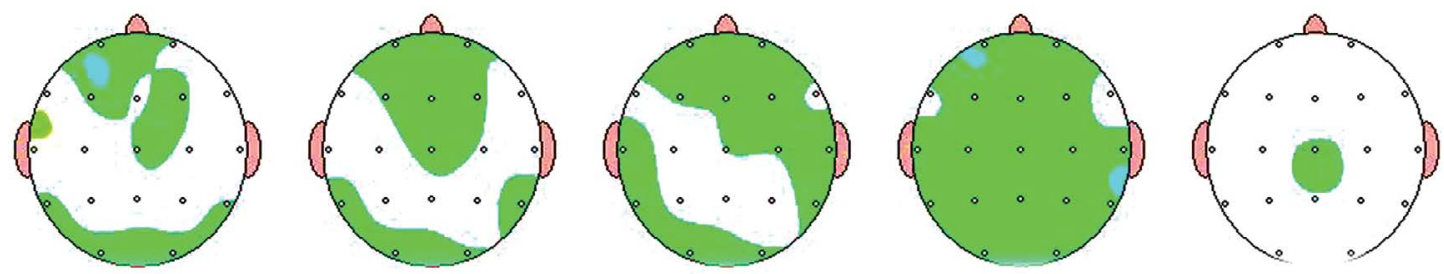

Pre

Delta

Theta

Alpha

Beta

High Beta

\section{Absolute Power}
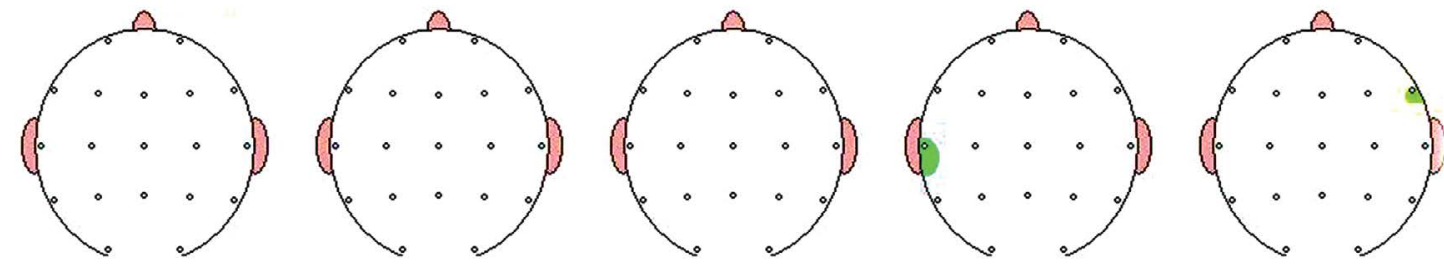

Post

Figure 1F: Subject 4 normalization in all frequencies across the spectrum after listening to The Magic Mirror CD once a day for 10 days.
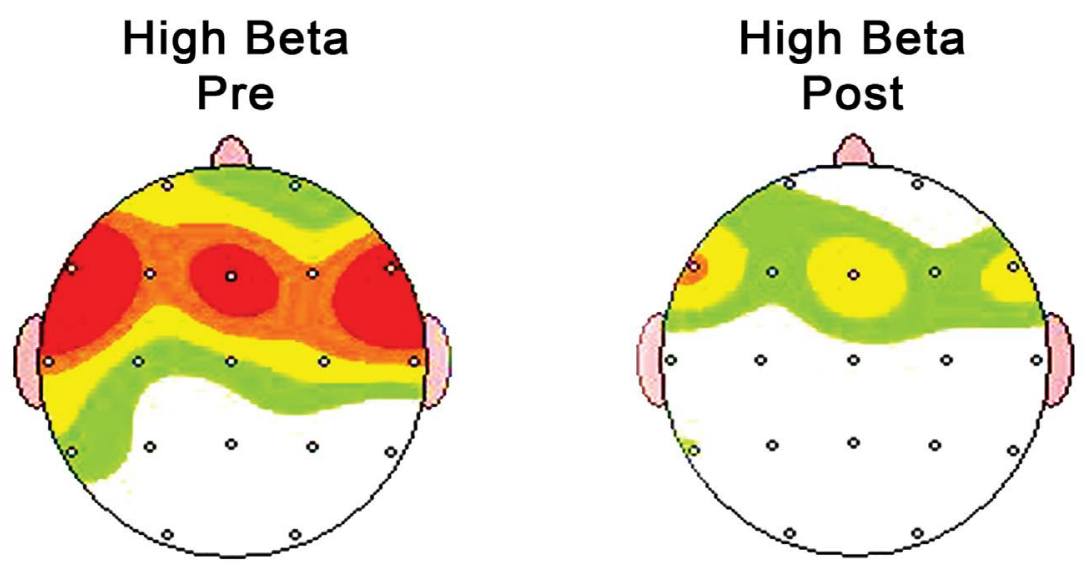

Figure 1G: Subject 5 decrease in high beta frequencies after listening to The Magic Mirror CD once a day for 10 days.

secretory IgA were from Sigma Chemical Co. Rabbit anti human CAVI was raised against affinity pure human salivary CAIV as described. Goat anti rabbit IgGperoxidase was from Sigma Chemical Co. Acrylamide and Bisacrylamide were obtained from Research Organics. Sodium dodecyl sulfate (SDS), bovine serum albumin (BSA), dithiothreitol (DTT), glycerol, and Folin Ciocalteu's phenol reagent were from Sigma Chemical Co. Biorad electrophoretic assembly, semi-dry transfer blot assembly, glass plates, and spacers were from Biorad Co. Immobilon-P membrane was purchased from Millipore. Luminol and 4-chlorophenol were from Aldrich Chemical Co. Autoradiography $\mathrm{X}$-ray film was purchased from Molecular Technologies, St. Louis, MO. All other reagents were of analytical grade.
Measurements of protein concentration in saliva: The frozen saliva samples were thawed on ice, centrifuged in table top eppendorf centrifuge at $16 \mathrm{~K}$ rpm for $10 \mathrm{~min}$ at $4{ }^{\circ} \mathrm{C}$. Fix volume, 2 ill of the saliva from each sample was used for protein determination. The protein concentration was determined by micro Lowry's procedure using bovine serum albumin (BSA) as a standard. In brief, 50 p.g of BSA in duplicate as a single point standard, or $2 \mathrm{pl}$ saliva $+48 \mathrm{~g} 11-120$, or $50 \mu \mathrm{l}$ $\mathrm{H}_{2} \mathrm{O}$ alone was incubated with $300 \mu \mathrm{l}$ of copper reagent freshly prepared by mixing 1 part $1 \%$ sodium dodecyl sulfate, 1 part 0.8 sodium hydroxide $(\mathrm{NaOH}), 1$ part $\mathrm{H}_{2} \mathrm{O}$, and 1 part of CTC solution $\left(0.1 \% \mathrm{CuSO}_{4} \cdot 5 \mathrm{H}_{2} \mathrm{O}+0.2 \% \mathrm{Na}-\mathrm{K}\right.$ tartarate. $4 \mathrm{H}_{2} \mathrm{O}$ in $5 \% \mathrm{Na}_{2} \mathrm{Co}_{3}$ ) and the reaction mixture was heated in a microwave for 10 seconds. The reaction 
mixture further mixed with 300 pi of Folin-Ciocalteu's phenol reagent and microwaved for an additional 10 seconds. The samples were cooled to room temperature and optical density at $750 \mathrm{~nm}$ was recorded on the spectrophotometer. The protein concentration in saliva was calculated from the ratio of optical density at 750 $\mathrm{nm}$ of 50 fig BSA over optical density at $750 \mathrm{~nm}$ of saliva sample. The maximum difference between duplicates was less than $4 \%$.

Preparation of sample for SDS-PAGE: Saliva samples containing 20-40 ug equivalent protein was mixed with equal volume of $2 \times$ SDS-PAGE sample buffer $(0.156 \mathrm{M}$ Tris HCL pH $6.8+5 \%$ SDS $+25 \% \mathrm{v} / \mathrm{v}$ glycerol and $50 \mathrm{mM}$ DTT) and heated at $98{ }^{\circ} \mathrm{C}$ for $1-2 \mathrm{mM}$. The SDS-PAGE samples were cooled to room temperature and stored at $-70{ }^{\circ} \mathrm{C}$. SDS-polyacrylamide gel electrophoresis: SDSPAGE was carried out according to Laemili's procedure. A $10 \%$ separating and $5 \%$ stacking acrylamide gel was cast in mini gel casting apparatus using $1.5 \mathrm{~nm}$ thick spacers. After samples were loaded to the gel, the protein polypeptides were separated by $40 \mathrm{~m}$ Amp constant current for $40 \mathrm{~min}$. The gel was recovered and prepared for electrophoretic transfer of the polypeptides to Immobilon-P membrane.

Western blot analysis: After electrophoresis, SDSPAGE gel was incubated in polypeptide transfer buffer,
$25 \mathrm{mM}$ Tris, $192 \mathrm{mM}$ glycine $+20 \%$ methanol for 20-30 $\mathrm{min}$. The polypeptides were transferred to Immobilon-P membrane using Trans Blot Semidry transfer gel apparatus (Biorad) at 10-12 volt per mini gel for $30 \mathrm{~min}$. The membrane was incubated with TBST $(10 \mathrm{mM}$ Tris- $\mathrm{HCL}$ $\mathrm{pH} 7.5+150 \mathrm{mM} \mathrm{NaCL}+0.05 \%$ Tween-20) $+1 \%$ casein for $20 \mathrm{mM}$ on shaking plate form at room temperature. The membrane was washed $3 \times 5 \mathrm{mM}$ each with $100-200$ $\mathrm{ml} \mathrm{TBST}$ by shaking and incubated with first antibodies, rabbit antihuman CAVI or 6-amylase or secretory IgA or IgG-FC at 1:3000-1:5000 dilutions at $4{ }^{\circ} \mathrm{C}$ overnight. The membrane was washed $4 \times 5$ min each with TBST and incubated with a second antibody, goat anti-rabbit IgG-peroxidase 1:3000-1:5000 dilutions at room temperature for $2 \mathrm{~h}$. Then the membrane was washed $4 \times 5$ min each with TBST and incubated with home-made luminol reagent for peroxidase at room temperature for 5 $\mathrm{min}$ in a plastic pouch. The substrate for peroxidase from the pouch was removed and the membrane was exposed to X-ray film for $10 \mathrm{sec}$ to $1 \mathrm{~min}$ and developed for the gel image. The western blot results were quantitated by densitometric recording of the polypeptide band intensities of X-ray film, using ImageQuant software.

\section{Results}

The results of pre - post qEEG recordings data analyzed by Dr. Robert Thatcher's normative database [22]

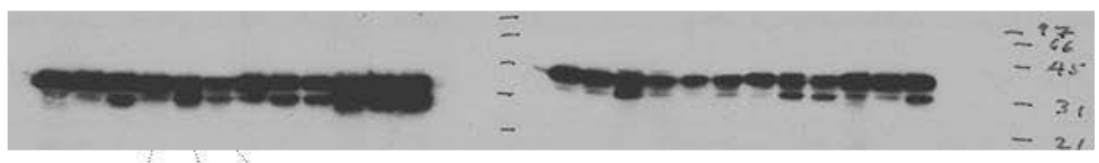

Carbonlc Anhydrase 6 (non-glycosylated)

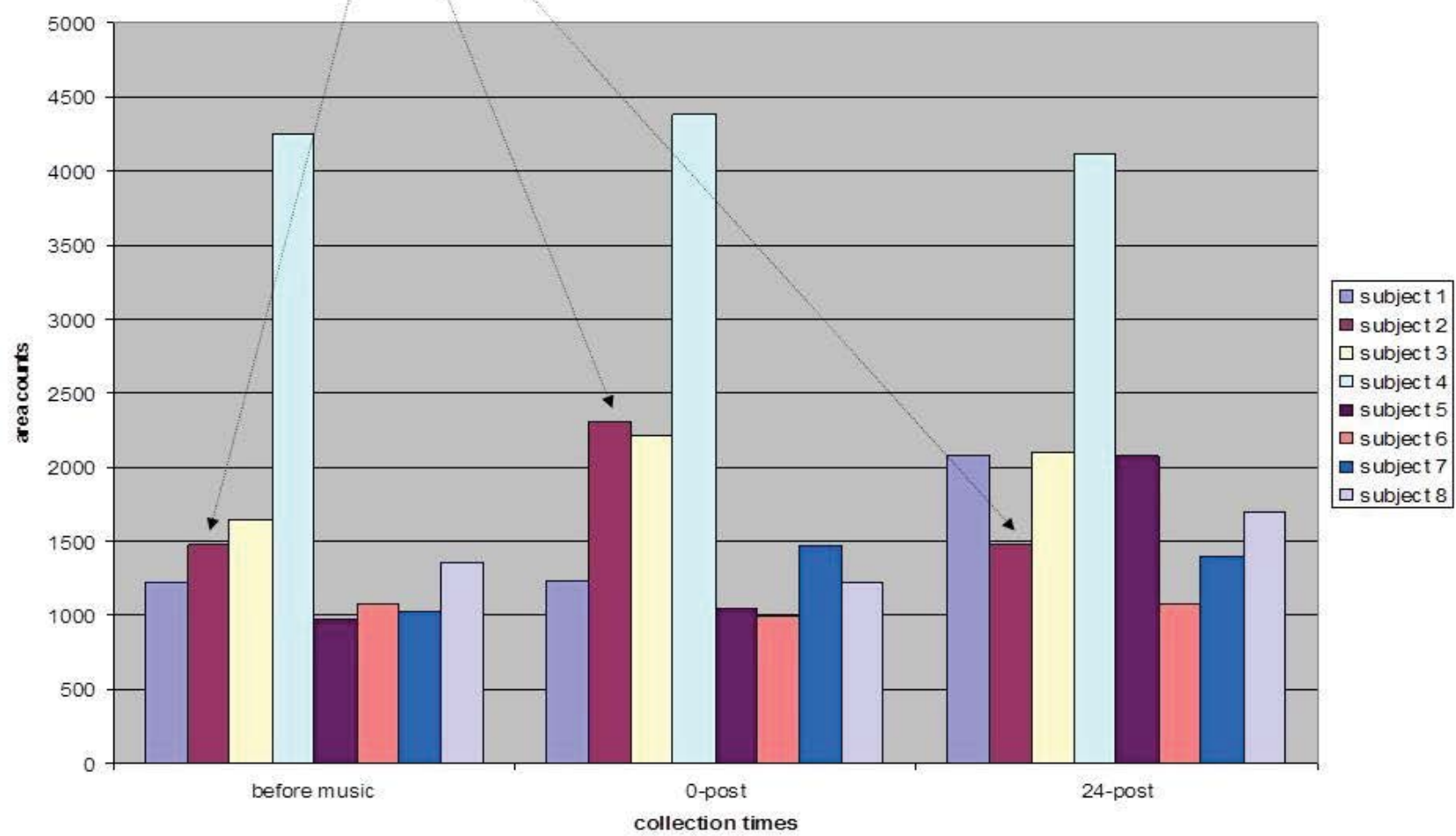

Figure 2: Carbonic anhydrase 6 (non-glycosylated) levels in saliva before listening to The Magic Mirror CD, immediately after listening, and 24 hours after listening. 
indicated significant changes in the EEG patterns of all 5 subjects. Each subject showed pre-study indications of EEG abnormalities and significant changes in the post qEEG recordings.

Subject 1: Pre qEEG indicated elevated delta, deficit theta, elevated beta, elevated high beta, no TBI index. Post qEEG indicated reduced delta, improved theta, normalized beta, reduced high beta, no TBI index.

Subject 2: Pre qEEG indicated elevated delta, elevated theta, deficit beta, elevated high beta, yes on TBI index. Post qEEG indicated reduced delta, normalized theta, improved beta, same high beta, no change TBI index.

Subject 3: Pre qEEG indicated elevated beta, elevated high beta, yes on TBI index. Post qEEG indicated reduced beta, reduced high beta, improved TBI index.

Subject 4: Pre qEEG indicated deficit theta, deficit beta, deficit high beta, yes on TBI index. Post qEEG indicated normalized theta, normalized beta, normalized high beta, improved TBI index.

Subject 5: Pre qEEG indicated deficit theta, deficit beta, elevated high beta, yes on TBI index. Post qEEG indicated normalized theta, normalized beta, reduced high beta, no change on TBI index.

Three of the five subjects showed a decrease in high beta. Two of the subjects showed a decrease in slow wave activity with one of those subjects showing a normalization of the EEG across the spectrum. The beta frequencies are considered fast waves and are used to process large amounts of information. The elevation of high beta is often associated with increased stress [27]. A decrease in these frequencies would therefore be associated with decreasing stress. The lower frequencies (delta and theta) are utilized by the brain to monitor internal processes. Elevations in these frequencies are often correlated with an increase in sympathetic nervous system arousal (stress) which has a negative impact on the immune system. A normalization of these frequencies also correlates with a decrease in stress which would have a positive impact on the immune system.

Daily Checklist questionnaires indicated all participants reported feeling better eight out of the ten days. Three of the five participants reported concentration and focus improved immediately after listening to the music.

\section{Salivary biomarkers}

In the first proof of principle pilot study with eight healthy individuals ranging in age from 8yrs to 65yrs, before and after listening to The Magic Mirror CD, we
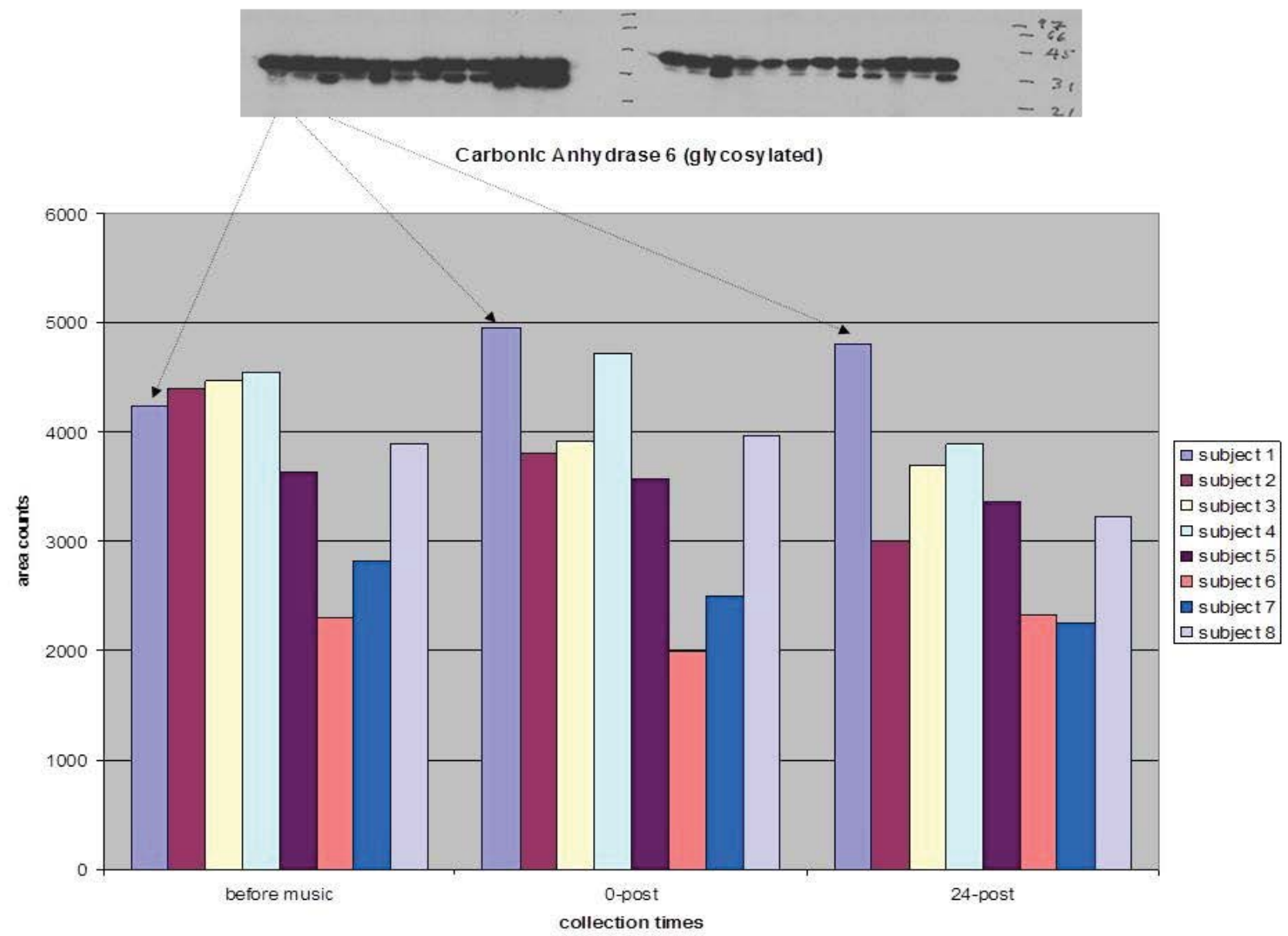

Figure 3: Carbonic anhydrase 6 (glycosylated) levels in saliva before listening to The Magic Mirror CD, immediately after listening, and 24 hours after listening. 


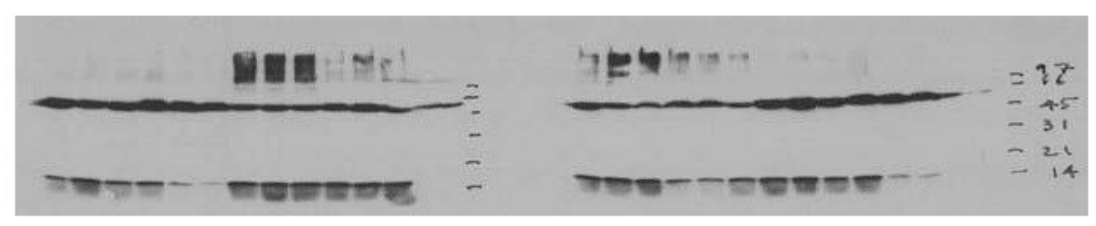

Amylase

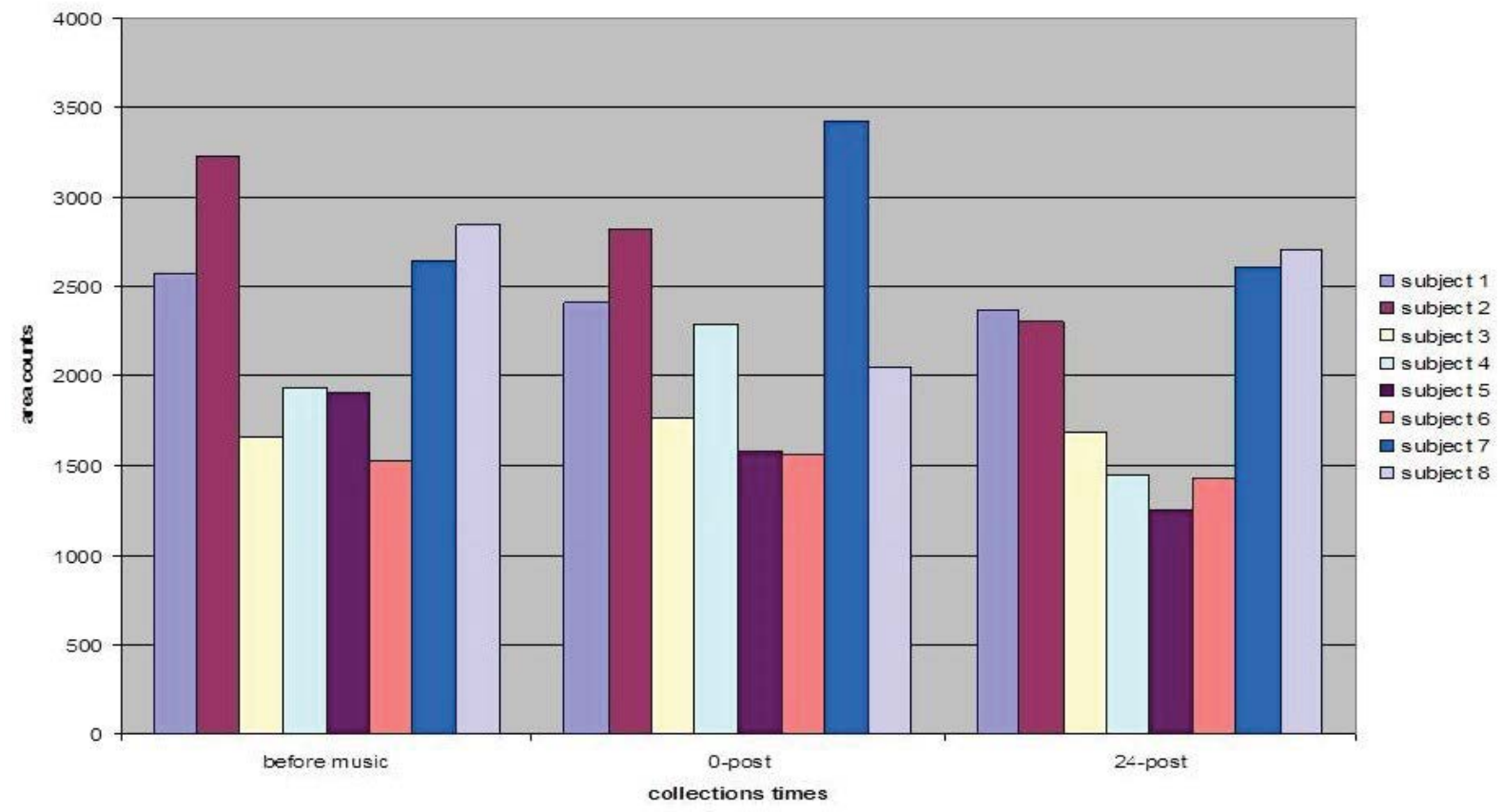

Figure 4: Amylase levels in saliva before listening to The Magic Mirror CD, immediately after listening, and 24 hours after listening.

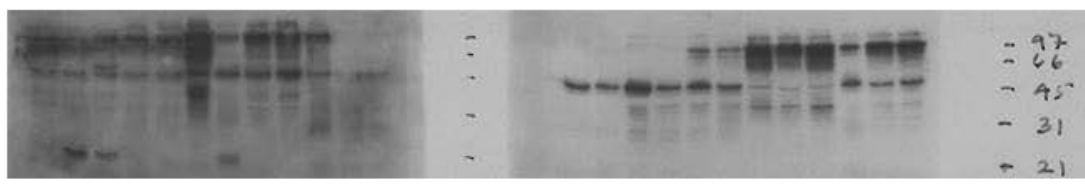

Hu lgG (heavy chain)

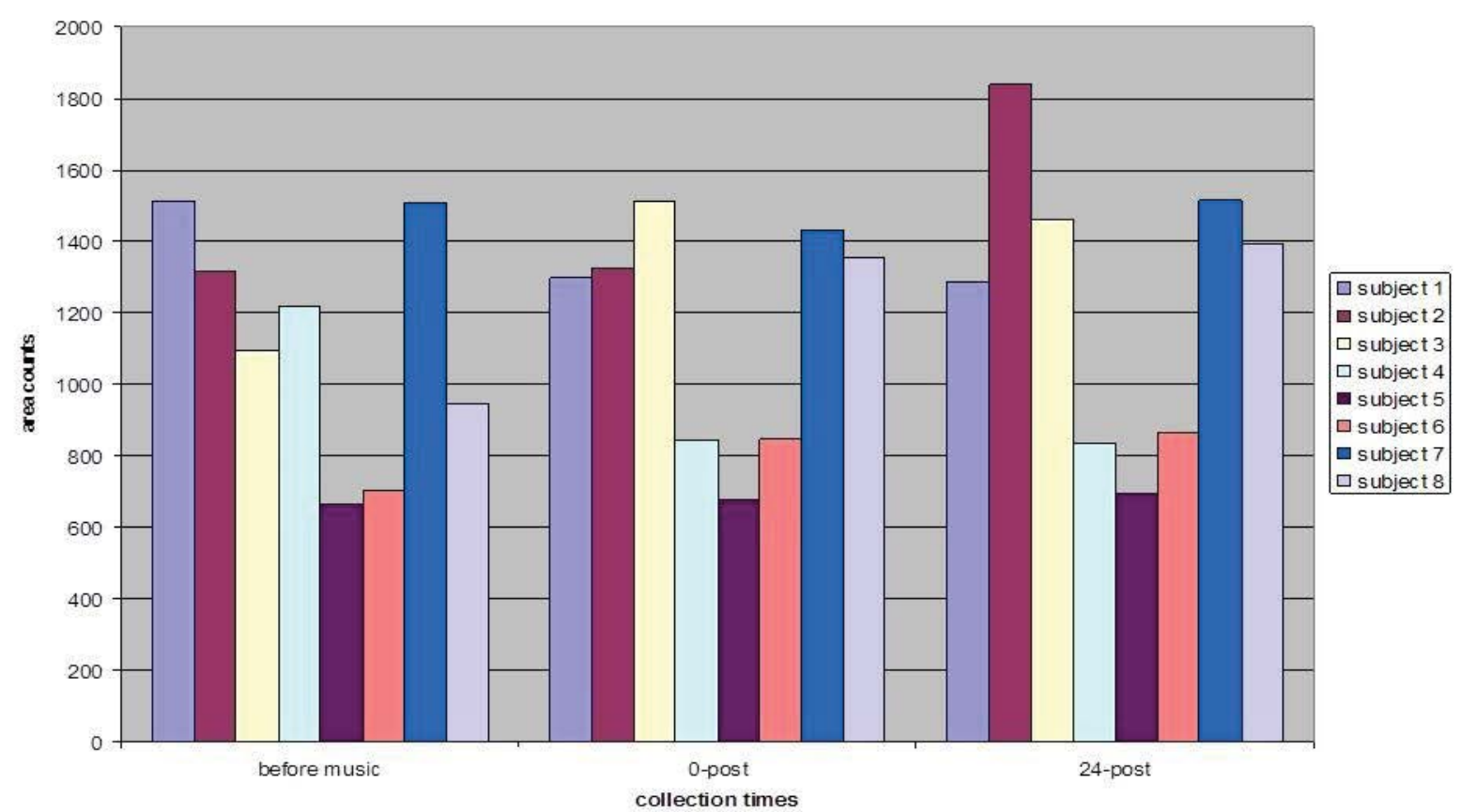

Figure 5: Hu IgG (heavy chain) levels in saliva before listening to The Magic Mirror CD, immediately after listening, and 24 hours after listening. 


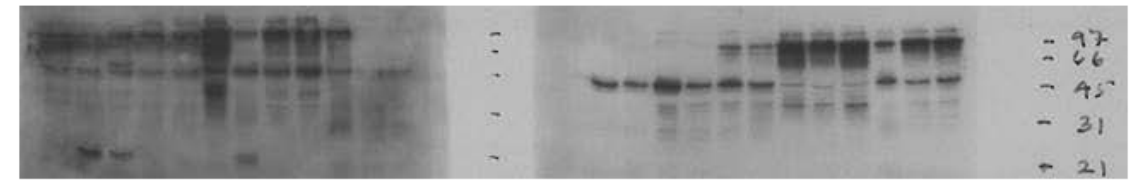

Hu lgG (light chain)

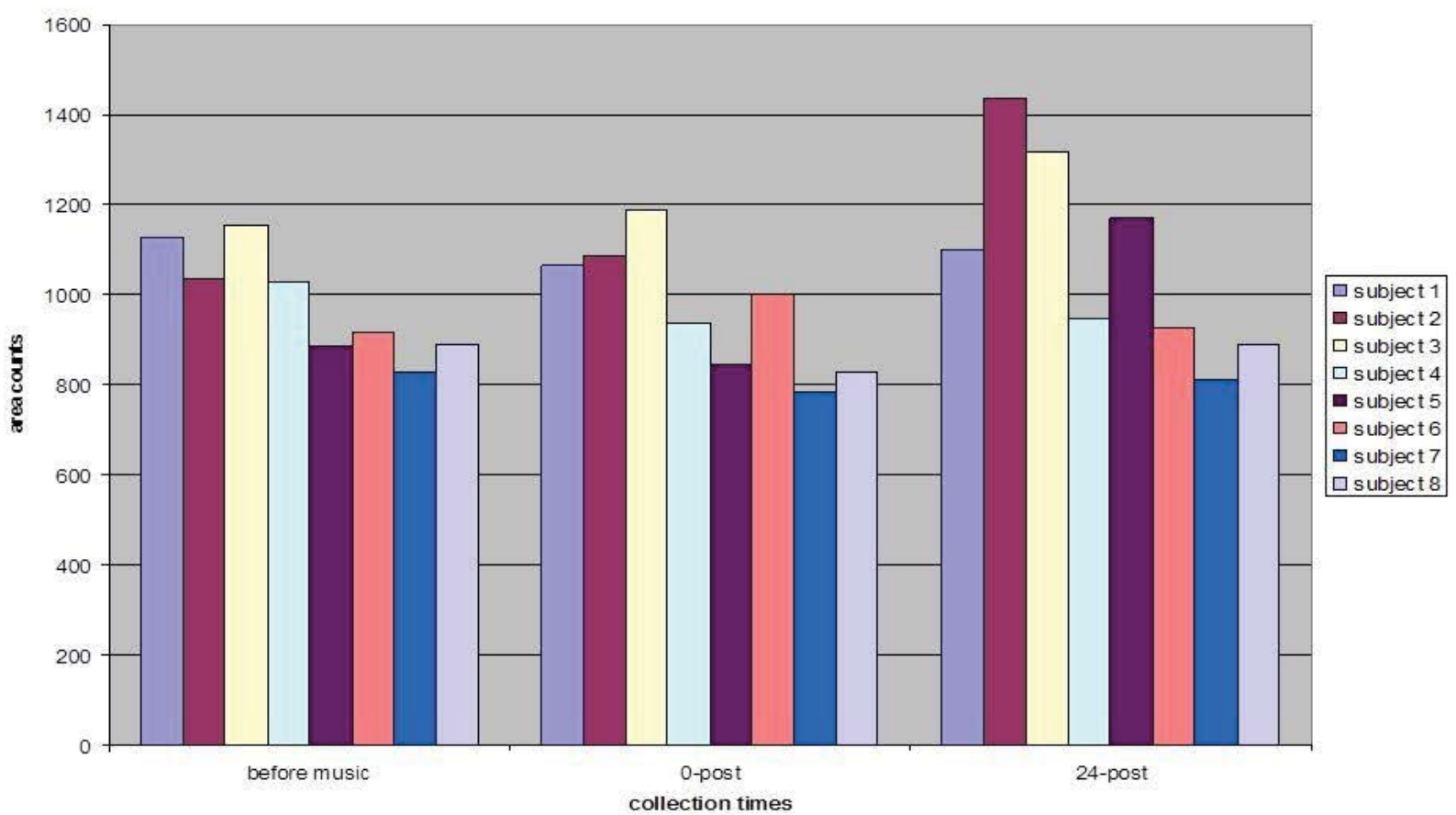

Figure 6: Hu IgG (light chain) levels in saliva before listening to The Magic Mirror CD, immediately after listening, and 24 hours after listening.

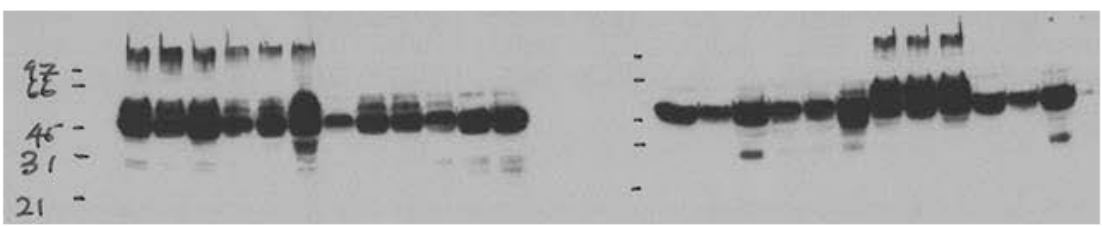

Hu SIgA (Lt chain; major band)

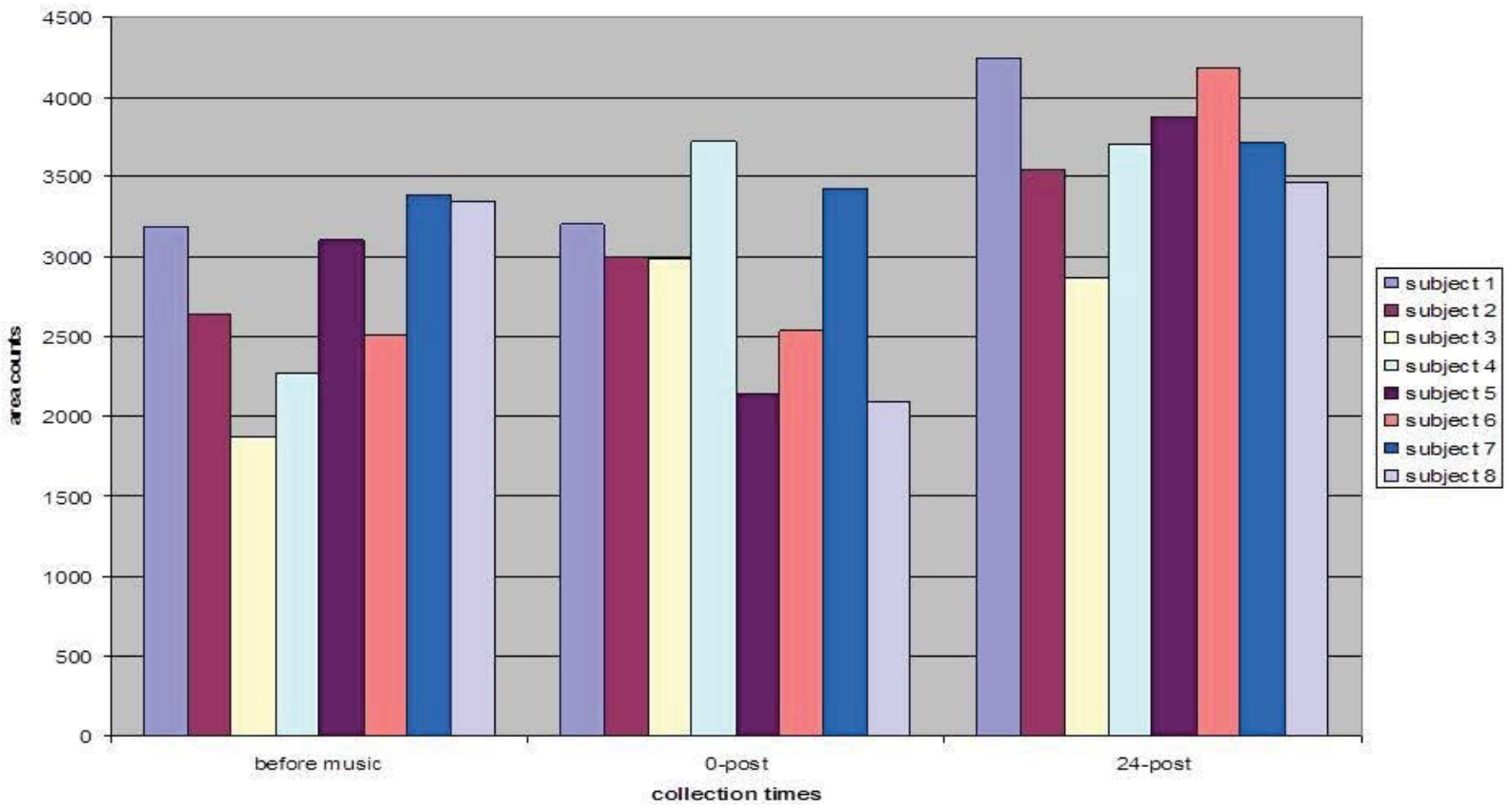

Figure 7: Hu SigA (Lt chain; major band) levels in saliva before listening to The Magic Mirror CD, immediately after listening, and 24 hours after listening. 


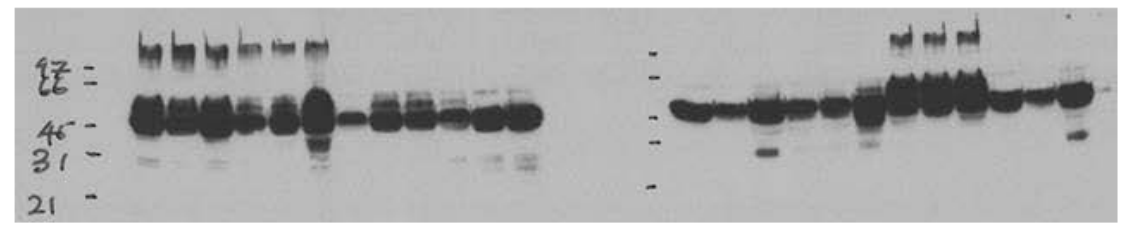

Hu SIgA (Lt chain; minor band)

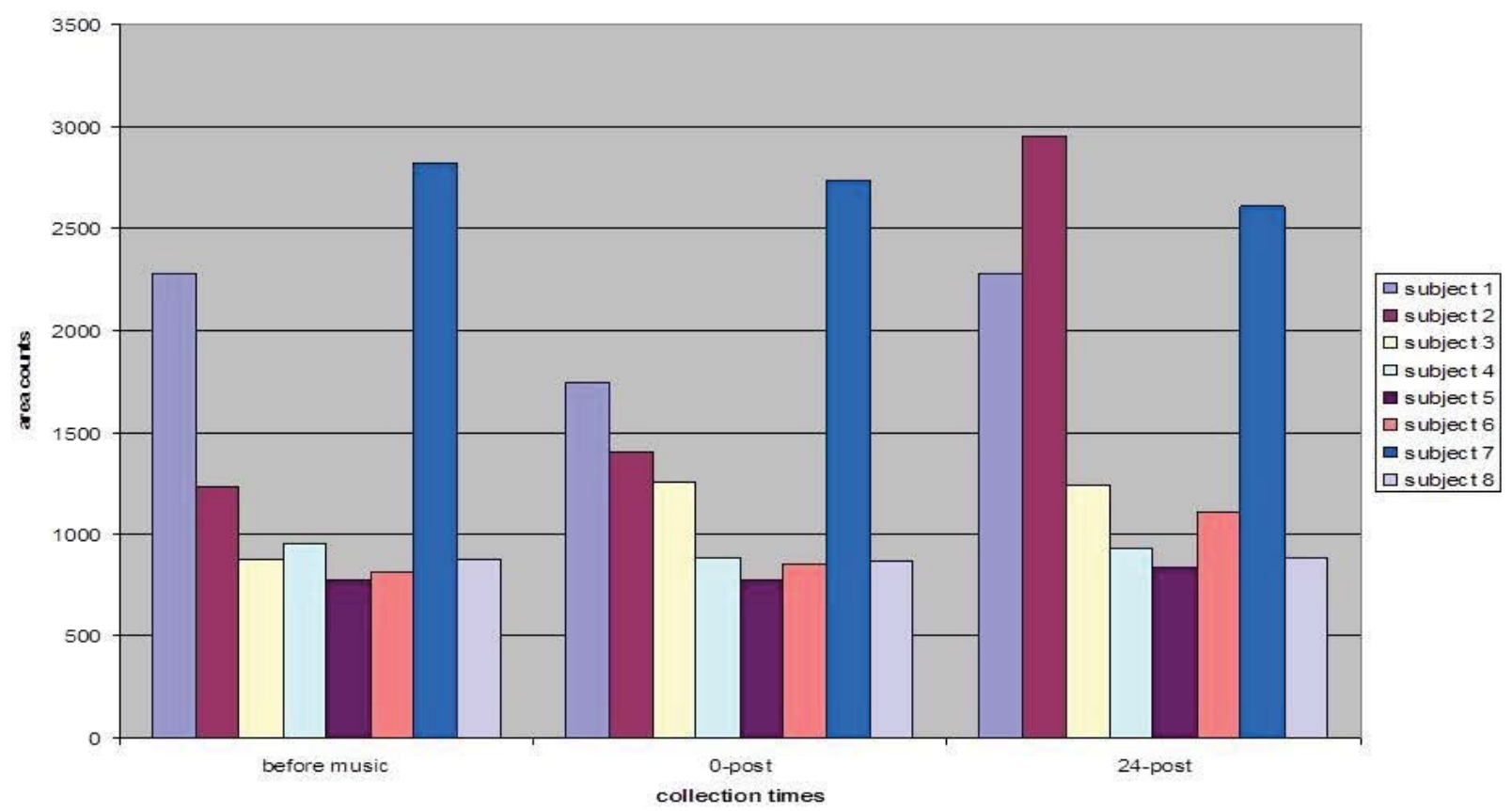

Figure 8: Hu SigA (Lt chain; minor band) levels in saliva before listening to The Magic Mirror CD, immediately after listening, and 24 hours after listening.

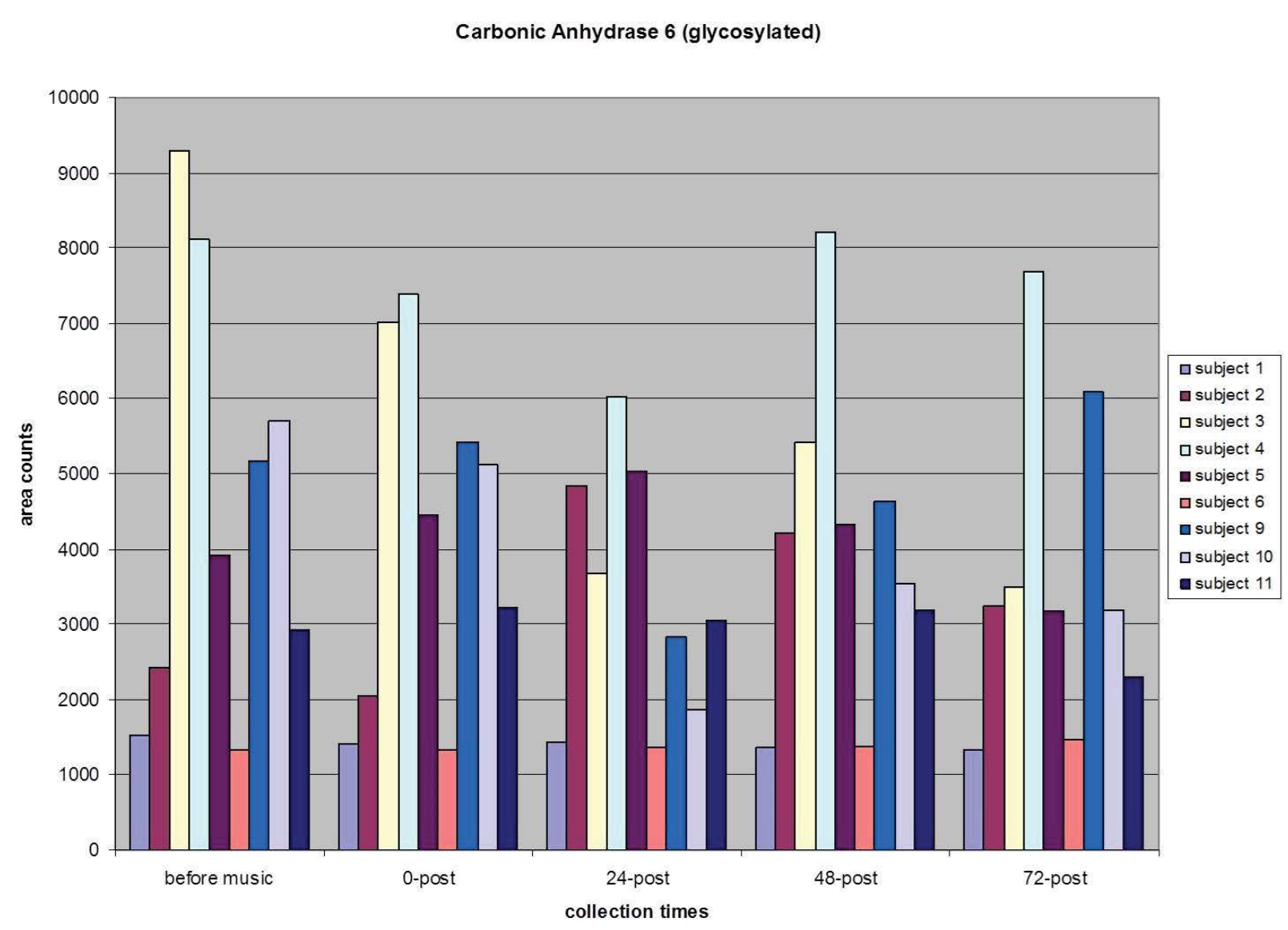

Figure 9: Carbonic anhydrase 6 (glycosylated) levels in saliva before listening to The Magic Mirror CD, immediately after listening, 24 hours, 48 hours, and 72 hours after listening. 


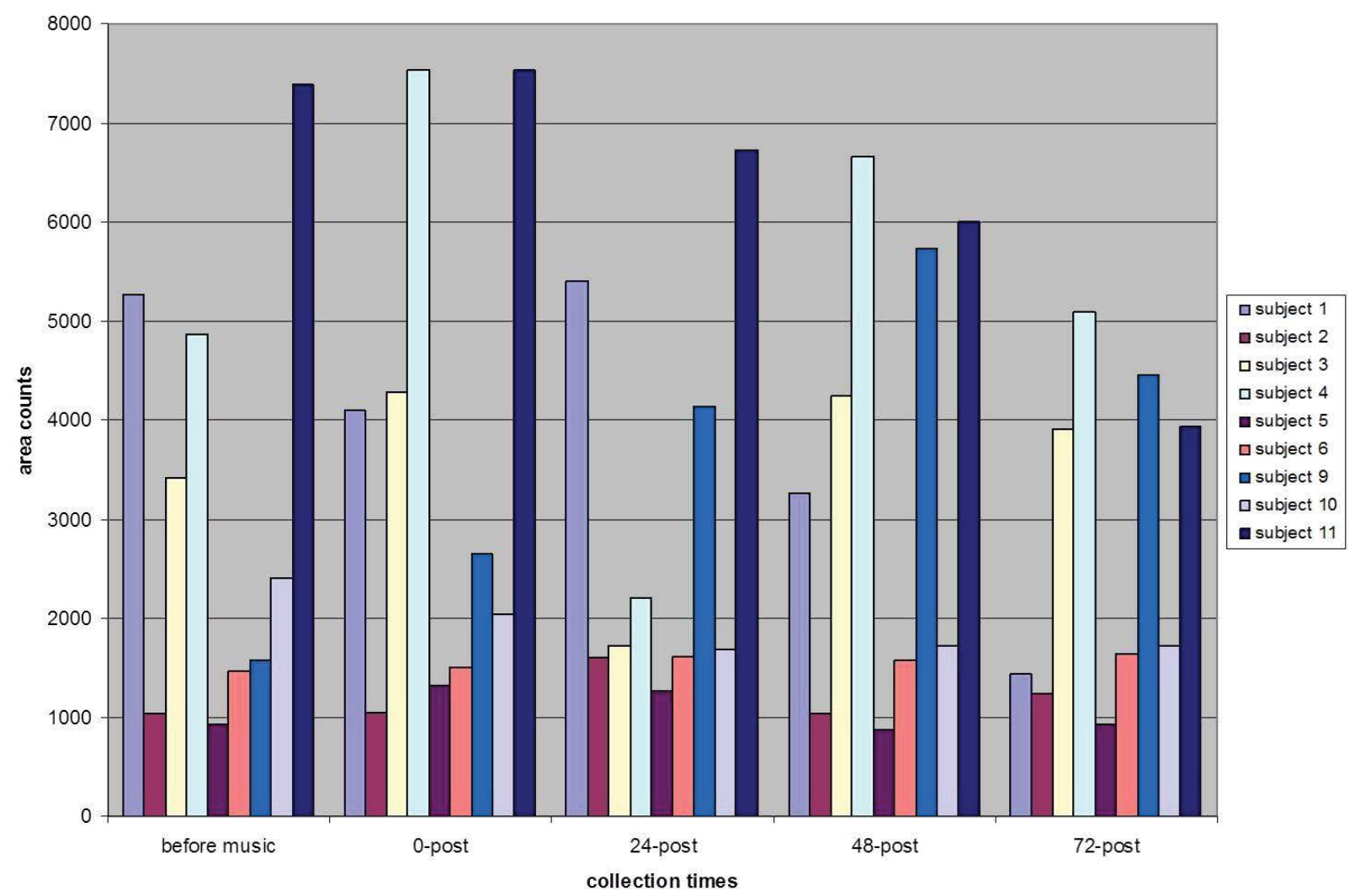

Figure 10: Carbonic anhydrase 6 (non-glycosylated) levels in saliva before listening to The Magic Mirror CD, immediately after listening, 24 hours, 48 hours, and 72 hours after listening.

\section{Human IgG (heavy chain)}

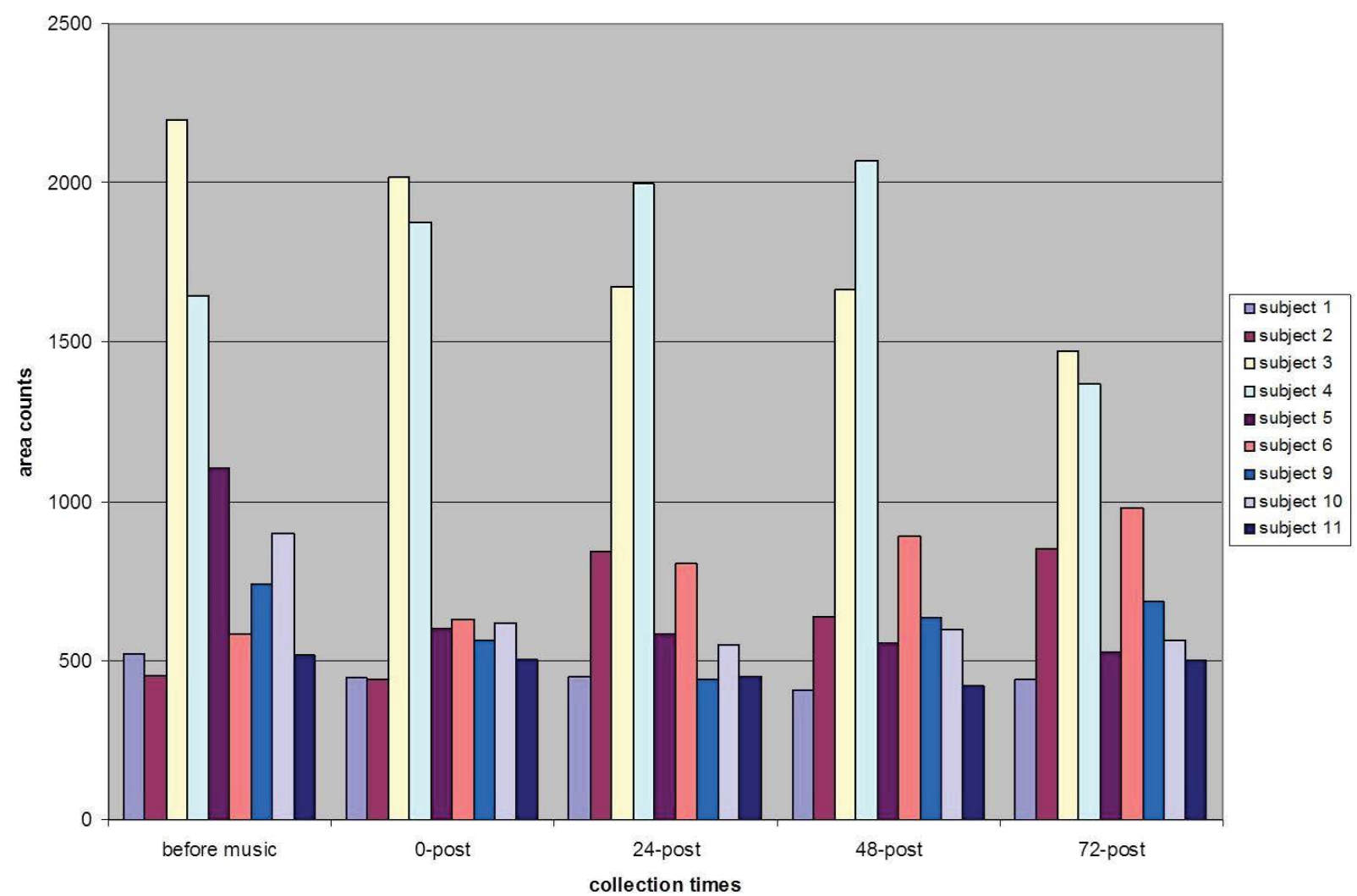

Figure 11: Human IgG (heavy chain) levels in saliva before listening to The Magic Mirror CD, immediately after listening, 24 hours, 48 hours, and 72 hours after listening. 


\section{Human IgG (light chain)}

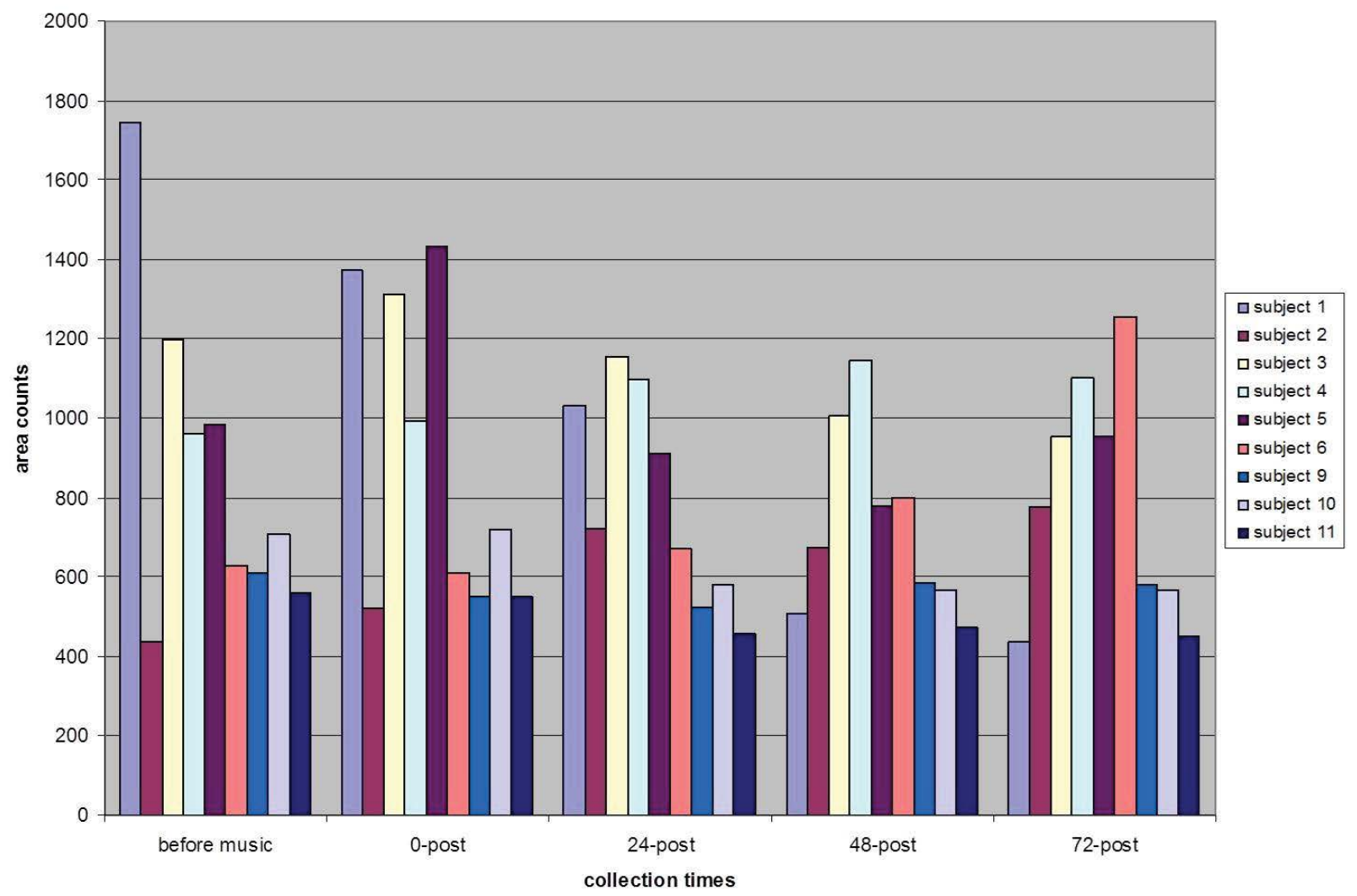

Figure 12: Human IgG (light chain) levels in saliva before listening to The Magic Mirror CD, immediately after listening, 24 hours, 48 hours, and 72 hours after listening.

\section{Human serum IgA (It chain major band)}

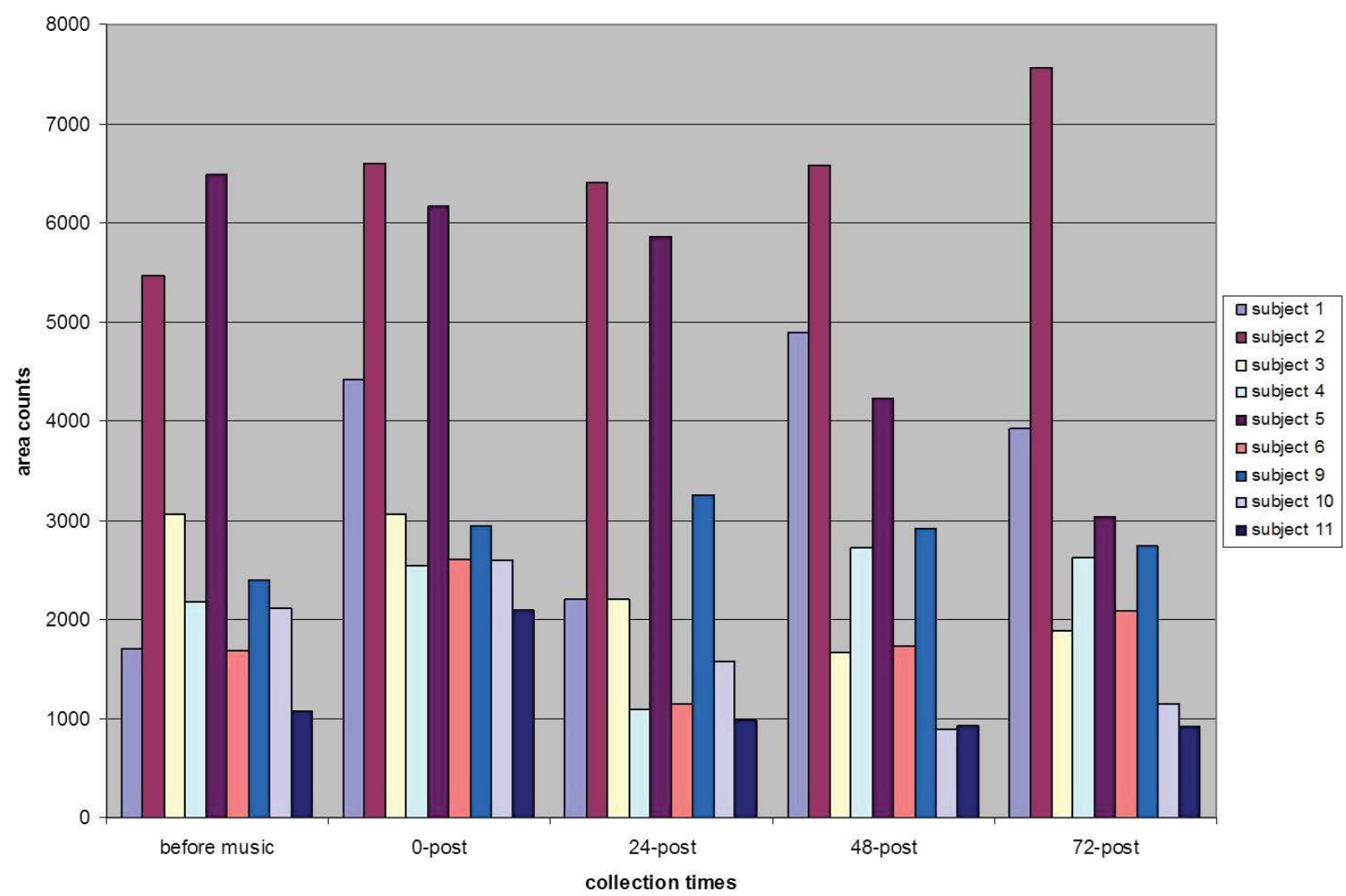

Figure 13: Human serum IgA (light chain major band) levels in saliva before listening to The Magic Mirror CD, immediately after listening, 24 hours, 48 hours, and 72 hours after listening. 
found that secretory $\lg A, \lg G$, and CAVI were increased and the increased level of these biomarkers remained high until 24 hours after music, suggesting that The Magic Mirror has a measureable effect on individuals by improving their immune parameters and function of salivary gland (Figure 2, Figure 3, Figure 4, Figure 5, Figure 6 , Figure 7 and Figure 8). Effect of music on d́-amylase secretion was not robust, but there was reproducible decrease in $\alpha$-amylase after music. These results are in accordance with literature where psychological stress has been found to increase $\alpha$-amylase secretion [28]. Therefore, relaxation effect of The Magic Mirror might have decreased the $\alpha$-amylase synthesis and secretion in saliva. It should be noted that few individuals responded better than others with The Magic Mirror. This result could be due to variation among individuals to respond to a specific music.

In the second pilot study, involving seven healthy individuals from the first study and two guest participants, we studied the duration of The Magic Mirror music effect on biomarkers. Similar to the first pilot study, levels of secretory IgA, IgG, $\dot{\alpha}$-amylase, and $\mathrm{CAVI}$ in saliva samples were increased post music and stayed high for 2-3 days (Figures 9, Figure 10, Figure 11, Figure 12, Figure 13 and Figure 14). Our two guest subjects were also responsive to the music. Their response of IgA and IgG towards music was relatively unstable compared to the other individuals. There were few normal individuals with poor response; however, response of music was sustained.

The results from these two proofs of principle pilot studies support the feasibility of utilizing carbonic anhydrase $\mathrm{VI}$ as a biomarker for stress.

\section{Discussion}

We analyzed the effect of specific music on cancer patients undergoing chemotherapy treatment. Results indicated a reduction of high beta waves which is an indicator of reduced stress. We analyzed saliva biomarkers of healthy individuals to determine the music's effect on immune system function. The study on healthy individuals helped us imagine the music we chose would have a beneficial effect on individuals going through cancer treatment.

More research is needed on effective ways to help cancer patients with the effects of chemo brain. Although the sample size was small, this study points to a possible adjunct treatment for cancer patients experiencing chemo brain symptoms and stress. The decrease in high beta waves and decrease in slow wave activity correlates to a decrease in stress. Subjective reports from all participants feeling better eight out of the ten days, and three participants reporting increased concentration and focus immediately after listening to the music, suggest listening to The Magic Mirror has a

Human serum IgA (It chain minor band)

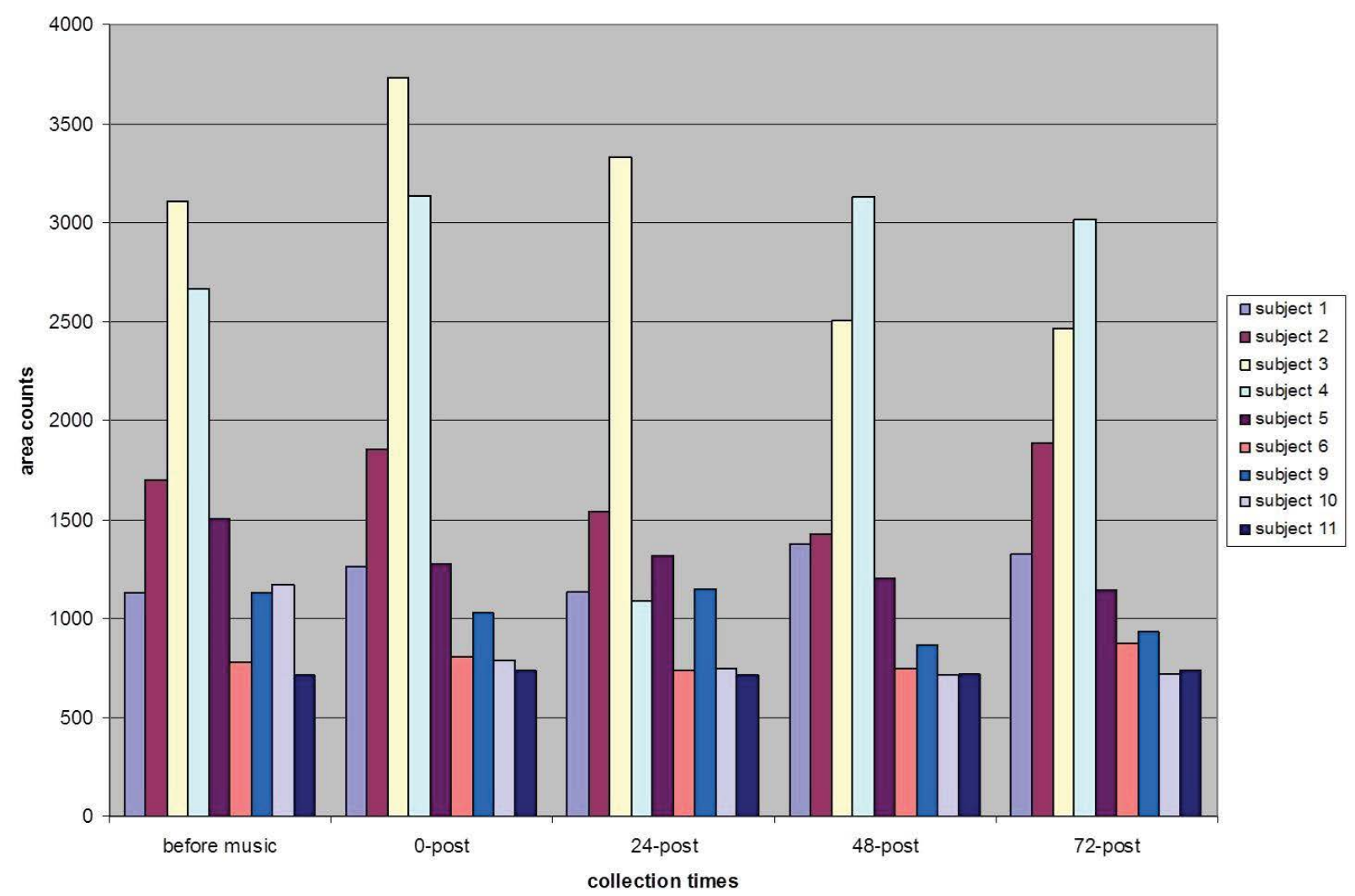

Figure 14: Human serum IgA (light chain minor band) levels in saliva before listening to The Magic Mirror CD, immediately after listening, 24 hours, 48 hours, and 72 hours after listening. 
positive impact on stress levels and symptoms relating to quality of life.

A larger study is needed to further explore the possibilities of The Magic Mirror solo harp music. Stress and its adverse effects on cancer patients can be further studied by looking at the long-term effects of The Magic Mirror on cancer treatment outcomes. A comparison of a course treatment while listening to The Magic Mirror and a control group that is not listening to The Magic Mirror would be such a study.

As a class, the carbonic anhydrases maintain ionic equilibria for cells, and also produce and maintain adequate bicarbonate reserves, as a component of the major buffering capacity of the body. With respect to carbonic anhydrase activity, all of the isozymes behave similarly. Since CAVI is the only carbonic anhydrase isozyme that is secreted from the cells, it also can perform as a signaling molecule between cells. With a labile zinc atom attached to each molecule, the CAVI is able to transfer its zinc "payload" to tissues that may require additional zinc, and in this way, the CAVI can act as a zinc chaperone, to facilitate the intercellular bioavailability of zinc. Since zinc is an essential component of over 300 extremely important zinc metalloenzymes, the health and maintenance of cells and tissues is critically dependent upon this facilitated delivery of adequate amounts of zinc in a predictable manner. Thus, the present study provides evidence that for certain individuals, a brief period of exposure to a specific piece of music is associated with an increased production of a critical intracellular zinc chaperone, that in turn, serves to support the health and maintenance of cells throughout the body. Importantly, these effects were observed to occur within minutes, and persisted for 2-3 days. Clearly, these data merit further investigations, to enable the full understanding of the therapeutic potential of The Magic Mirror music in a variety of disease states.

The variability in the qEEG and salivary biomarker results could be related to the health and age of each subject. Variations could also be related to the intention of the composer/performer of the music, resulting in each listener receiving individualized benefit from the music.

Admittedly, the small numbers in these three sample studies do not enable a statistical analysis, but trends do appear that merit further investigations. A larger study is needed to explore the effects of The Magic Mirror solo harp music on immune system function, chemo brain, and stress.

\section{Conclusion}

The indications that can be drawn from the qEEG pilot study are:

1. The Magic Mirror solo harp CD has a significant effect on EEG frequencies of the subjects in the study.

2. The frequencies most affected are the High Beta and Theta.

A general comment that can be made is The Magic Mirror solo harp CD calms and quiets the brain resulting in greater ability to focus and process information.

Future studies can look at the impact of The Magic Mirror-Inspired Reflections solo harp CD to determine if there is a direct impact on chemo brain.

The data also suggest that during and after listening to The Magic Mirror, a substantial number of respondents also experienced neurosecretory responses that were associated with the release of immunomodulatory cytokines. These signaling molecules play divergent roles in maintaining the health and wellbeing of the individual. In our IgG and IgA biomarker studies, some individuals had an elevated response to music which suggests this music not only has a benefit on the human brain but also influences the immune response. There is evidence that increasing IgA protects the individual from infection [29].

Thus, the results of these studies suggest that listening to The Magic Mirror solo harp CD can be used in combination with other therapy for cancer or physiologically stressed patients to reduce the magnitude and duration of the effect of their disease.

\section{Acknowledgements}

The authors of the manuscript are grateful for the participants of this study.

The Scientific Arts Foundation provided administrative support and funds to implement the qEEG analysis. Chris Collins provided administrative support to William Collins.

John Camie provided inspiration and production expertise for the recording of The Magic Mirror-Inspired Reflections CD.

Kristy Shaughnessy provided proofreading services.

A special thanks to St. Louis University Department of Biochemistry and Molecular Biology for all biochemical studies. One of us, AW, acknowledges the help from Professor W.S. Sly.

\section{Conflict of Interest}

None.

\section{Authorship Contributions}

Abdul Waheed and David Kossor conceived, developed, and implemented the saliva sample study design, completed the analysis and interpretation of data, and contributed to the final manuscript.

William Collins conceived and developed the qEEG study design, collected raw data, completed analysis 
and interpretation of data, and contributed to the final manuscript.

Amy Camie wrote and recorded The Magic Mirror - Inspired Reflections CD and contributed to the final manuscript.

\section{References}

1. Martin Saavedra J, Vergara Mendez L, Pradilla I, Vélez van Meerbeke A, Talero Gutiérrez C (2018) Standardizing music characteristics for the management of pain: A systematic review and meta-analysis of clinical trials. Complementary Therapies in Medicine 41: 81-89.

2. European Society of Cardiology (2018) Listening to yoga music at bedtime is good for the heart. Science Daily.

3. Bernardi L, Porta C, Casucci G, Balsamo R, Bernardi $\mathrm{N}$, et al. (2009) Dynamic interactions between musical, cardiovascular, and cerebral rhythms in humans. Circulation 119: 3171-3180.

4. Gabrielsson A, Juslin PN (1996) Emotional expression in music performance: Between the performer's intention and the listener's experience. Psychology of Music 24: 68-91.

5. Bear MF, Connors BW, Paradiso MA (1996) Neuroscience: Exploring the brain. Williams and Wilkins, Baltimore, MD, USA.

6. Van der Pompe G, Antoni MH, Heijnen CJ (1996) Elevated basal cortisol levels and attenuated ACTH and cortisol responses to a behavioral challenge in women with metastatic breast cancer. Psychoneuroendocrinology 21: 361-374.

7. Gruess DG, Antoni MH, McGregor BA, Kilbourn KM, Boyers $A E$, et al. (2000) Cognitive-behavioral stress management reduces serum cortisol by enhancing benefit finding among women being treated for early stage breast cancer. Psychosom Med 62: 304-308.

8. Gruess DG, Antoni MH, Kumar M, Schneiderman N (2000) Reduction in salivary cortisol are associated with mood improvement during relaxation training among HIVseropositive men. J Behav Med 23: 107-122.

9. Seo SH, Lee JT (2010) Stress and EEG. In Convergence and hybrid information technologies. InTech.

10. Cohen S, Janicki Deverts D, Miller GE (2007) Psychological stress and disease. JAMA 298: 1685-1687.

11. Jean Pierre P, Johnson Greene D, Burish TG (2014) Neuropsychological care and rehabilitation of cancer patients with chemobrain: Strategies for evaluation and intervention development. Support Care Cancer 22: 22512260.

12. Koppelmans V, Breteler MB, Boogerd W, Seynaeve C, Gundy C, et al. (2012) Neuropsychological performance in survivors of breast cancer more than 20 years after adjuvant chemotherapy. J Clin Oncol 30: 1080-1086.
13. Bradt J, Potvin N, Kesslick A, Minjung Shim, Donna Radl, et al. (2015) The impact of music therapy versus music medicine on psychological outcomes and pain in cancer patients: A mixed methods study. Support Care Cancer 23: 1261-1271.

14. Wolters Kluwer Health (2014) Cancer nursing: An international journal for cancer care 37 .

15. Fancourt D, Ockelford A, Belai A (2014) The psychoneuroimmunological effects of music: A systematic review and a new model. Brain Behav Immun 36: 15-26.

16. National Institute of Health (NIH) (2018) Side effects of cancer treatment.

17. Soo Quee Koh D, Choon Huat Koh G (2007) The use of salivary biomarkers in occupational and environmental medicine. Occup Environ Med 64: 202-210.

18. Kivela, J, Parkkila S, Parkkila AK, Leinonen J, Rajaniemi H (1999) Salivary carbonic anhydrase isoenzyme VI. J Physiol 520: 315-320.

19. Calò C, Padiglia A, Zonza A, Corrias L, Contu P, et al. (2011) Polymorphisms in TAS2R38 and the taste bud trophic factor, gustin gene co-operate in modulating PROP taste phenotype. Physiology \& Behavior 104: 1065-1071.

20. Thatcher B, Doherty A, Orvisky E, Martin B, Henkin R (1998) Gustin from human parotid saliva is carbonic anhydrase VI. Biochemical and Biophysical Research Communications 250: 635-641.

21. http://www.amycamie.com/qeeg-pilot-study-results.html

22. Thatcher RW, Biver CL, North D, Curtin R, Walker RW (2003) Quantitative EEG normative databases: Validation and clinical correlation. Journal of Neurotherapy 7: 87-121.

23. McClelland DC, Ross G, Patel V (1985) The effect of an academic examination on salivary norepinephrine and immunoglobulin levels. J Human Stress 52-59.

24. Glaser R, Kiecolt Glaser JK, Speicher CE, Holliday JE (1985) Stress, loneliness, and changes in herpesvirus latency. J Behav Med 8: 249-260.

25. Takai N, Yamaguchi M, Aragaki T, Eto K, Uchihashi K, et al. (2004) Effect of psychological stress on the salivary cortisol and amylase levels in healthy young adults. Arch Oral Biol 49: 963-968.

26. Leinonen J, Kivela J, Parkkila S, Parkkila AK, Rajaniemi H (1999) Salivary carbonic anhydrase isozyme VI is located in human enamel. Caries Res 33: 185-190.

27. Jena SK (2015) Examination stress and its effect on EEG. Int J Med Sci Public Health 4: 1493-1497.

28. Van Stegeren A, Rohleder N, Everaerd W, Wolf OT (2006) Salivary alpha amylase as marker for adrenergic activity during stress: Effect of beta blockade. Psychoneuroendocrinology 31: 137-141.

29. Janeway CA Jr, Travers P, Walport M (2001) Immunobiology: The Immune System in Health and Disease. $\left(5^{\text {th }}\right.$ edn $)$, Garland Science, New York. 